\section{KOMPASS}

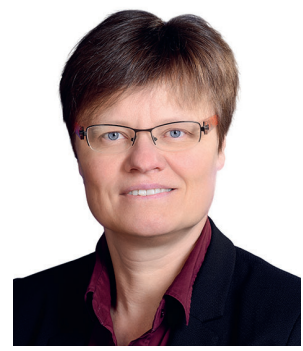

Susanne Saußele

III. Medizinische Klinik Hämatologie und Internistische Onkologie, Universitätsmedizin Mannheim GmbH, Mannheim, Deutschland

\title{
Die CML geht voran
}

Wir haben es weit gebracht in der Therapie der chronischen myeloischen Leukämie. Mit der Einführung der Tyrosinkinaseinhibitoren (TKI) kann die Erkrankung so effektiv behandelt werden, dass viele Patienten eine normale Lebenserwartung haben. Unter bestimmten Bedingungen kann die TKI-Therapie auch wieder abgesetzt werden - nach 3 Jahren Therapie und mindestens einem Jahr dauerhaft tiefer molekularer Remission (DMR) ist das in Deutschland seit 2012 im Rahmen von Studien möglich. Sogar die therapiefreie Remission (TFR), die Chance, dauerhaft therapiefrei zu bleiben, besteht für CMLPatienten heute. Eine bis vor 10 Jahren noch undenkbare Entwicklung für diese Erkrankung und einmalig bisher in der Onkologie.

Sind damit die Probleme in der Behandlung der CML gelöst? Natürlich nicht. Immer noch gibt es Patienten, die nicht ausreichend auf die Therapie ansprechen und eine primäre oder sekundäre Resistenz haben bzw. entwickeln. Unter Erstlinientherapie trifft das auf 10-15\% der Patienten zu, unter dem TKI der ersten Generation Imatinib auf bis zu 20\%. In der Praxis werden über 40\% der Patienten auf eine Zweitlinienoder gar Drittlinientherapie umgestellt. Nach aktuellen Zahlen erreichen nur etwa 20\% der CML-Patienten eine TFR.
Auch hinsichtlich des Monitorings gibt es noch offene Fragen: Brauchen wir Next-Generation Sequencing (NGS) zur Mutationsanalyse? Ist die biologische Wertigkeit des Transkripttyps des BCR-ABL-Bruchpunktes unterschiedlich?

Und schlussendlich steht immer die Frage, wie der Anteil der Patienten in TFR erhöht werden kann. Brauchen wir dazu bessere Erstlinien-Strategien? Oder ist es ausreichend, mit Imatinib zu beginnen und erst die Therapie zu wechseln, wenn keine DMR erreicht wird? Welches sind hier die entscheidenden Zeitpunkte, wann ist der richtige Zeitpunkt zur allogenen Stammzelltransplantation gekommen? Fragen zur Lebensqualität unter TKI in TFR nach Stammzelltransplantation sind bisher überhaupt wenig erforscht.

Es gibt also noch viel zu tun und dies sind auch nur einige der Fragen, die es weiterhin zu klären gibt. Aber wir sind dran, wie auch einige der Kommentare in der Ihnen nun vorliegenden Ausgabe des KARGER KOMPASS ONKOLOGIE zum Schwerpunkt CML zeigen. Ich wünsche Ihnen also eine anregende Lektüre

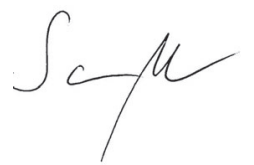

Ihre Susanne Saußele

\section{KARGER}

Fax +497614520714 information@karger.com www.karger.com

\section{() 2019 S.Karger GmbH, Freiburg}

Prof. Dr. Susanne Saußele

III. Medizinische Klinik für Hämatologie und Onkologie

Universitätsklinikum Mannheim

Theodor-Kutzer-Ufer 1-3, 68167 Mannheim, Deutschland

susanne.saussele@medma.uni-heidelberg.de 